\title{
ARTICLE Real-time simultaneous recording of electrophysiological activities and dopamine overflow in the deep brain nuclei of a non-human primate with Parkinson's disease using nano-based microelectrode arrays
}

\author{
Song Zhang ${ }^{1,2}$, Yilin Song ${ }^{1,2}$, Mixia Wang ${ }^{1,2}$, Guihua Xiao ${ }^{1,2}$, Fei Gao ${ }^{1,2}$, Ziyue $\mathrm{Li}^{1,2}$, Guoxian Tao ${ }^{3}$, Ping Zhuang ${ }^{4}$, Feng Yue ${ }^{3,4}$, \\ Piu Chan ${ }^{4}$ and Xinxia Cai ${ }^{1,2}$
}

Parkinson's disease (PD) is characterized by a progressive degeneration of nigrostriatal dopaminergic neurons. The precise mechanisms are still unknown. Since the neuronal communications are inherently electrical and chemical in nature, dual-mode detection of PD-related neuroelectrical and neurochemical information is essential for PD research. Subthalamic nucleus (STN) highfrequency stimulation (HFS) can improve most symptoms of PD patients and decrease the dosage of antiparkinsonian drugs. The mechanism of STN-HFS for PD still remains elusive. In this study, a silicon-based dual-mode microelectrode array (MEA) probe was designed and fabricated, and systematic dual-mode detection methods were established. The recording sites were modified using Pt nanoparticles and Nafion to improve the signal-to-noise (SNR) ratio. To evaluate its applicability to PD research, in vivo electrophysiological and electrochemical detection was performed in normal and hemiparkinsonian models, respectively. Through comparison of the dual-mode signals, we demonstrated the following in a PD monkey: (1) the maximum dopamine concentration in the striatum decreased by $90 \%$; (2) the spike firing frequency increased significantly, especially in the region of the cortex; (3) the spectrogram analysis showed that much power existed in the $0-10 \mathrm{~Hz}$ frequency band; and (4) following repeated subthalamic nucleus high-frequency stimulation trials, the level of DA in the striatum increased by $16.5 \mu \mathrm{m}$, which led to a better elucidation of the mechanism of HFS. The dual-mode MEA probe was demonstrated to be an effective tool for the study of neurological disorders.

Keywords: dual mode; implantable MEA; non-human primate; Parkinson's disease; STN-HFS

Microsystems \& Nanoengineering (2018) 4, 17070; doi:10.1038/micronano.2017.70; Published online: 1 January 2018

\section{INTRODUCTION}

Parkinson's disease (PD) is a progressive neurodegenerative illness that involves the following motor symptoms: tremor, bradykinesia, and rigidity. The disease weakens the self-care ability and greatly reduces the life quality of patients. Unfortunately, the precise mechanisms responsible for PD are still unclear ${ }^{1,2}$. At present, the most effective experimental model of PD is a 1-methyl-4-phenyl1,2,3,6-tetrahydropyridine (MPTP)-treated primate. The non-human primate animal models induced by MPTP can reflect the fundamental characteristics of PD and the response to all commonly used antiparkinsonian agents and are considered to be some of the best animal models regarding the similarity to human beings ${ }^{3-5}$.

Over the years, many attempts have been made to improve people's understanding of PD. In parkinsonian patients and animal models, local field potentials and neural spikes, which demonstrate the existence of synchronized oscillatory activity within neuronal populations of the basal ganglia and motor cortex, have been recorded $^{6,7}$. Furthermore, dopamine (DA) is a key neurotransmitter related to the development of PD. The available physiological and anatomical studies strongly suggest that the pathologic feature of this disease is the progressive degeneration of nigral dopamine neurons, which leads to severe dopaminergic denervation of the corpus striatum. Morphological and neurochemical studies have found the marked and consistent reduction of the DA concentration in the striatum of PD patients ${ }^{8,9}$. As we know, in vivo extracellular DA contents are in the low nanomolar level. The technical difficulties of detection tools have prevented the detailed examination of extracellular DA regulation ${ }^{10,11}$. Therefore, our knowledge about the nature of DA neural transmission and the overall regulation of extracellular DA levels is quite limited.

Currently, PD is most effectively treated using medications, such as levodopa. The drug levodopa can increase the DA level in the basal ganglia and alleviate the symptoms of PD. However, long-term use of these drugs will cause severe side effects, such as motor fluctuations and dyskinetic movement abnormalities ${ }^{12,13}$. Subthalamic nucleus (STN) high-frequency stimulation (HFS) can improve most symptoms of PD patients and decrease the dosage of antiparkinsonian, drugs such as Madopar ${ }^{14}$. The mechanism of STN-HFS for PD still remains elusive. Researchers have hypothesized that the level of DA and its metabolites in the corpus striatum are increased after STN-HFS.

\footnotetext{
${ }^{1}$ State Key Laboratory of Transducer Technology, Institute of Electronics, Chinese Academy of Sciences, Beijing 100190, China; ${ }^{2}$ University of Chinese Academy of Sciences, Beijing 100190, China; ${ }^{3}$ Wincon TheraCells Biotechnologies Co., Ltd., Nanning 530002, China and ${ }^{4}$ Department of Neurobiology, Xuanwu Hospital, Capital Medical University, The Beijing Key Laboratory of Parkinson's Disease, Beijing 100053, China.

Correspondence: Feng Yue (fyuee@hotmail.com) or Piu Chan (pbchan90@gmail.com) or Xinxia Cai (xxcai@mail.ie.ac.cn)

Received: 1 April 2017; revised: 18 July 2017; accepted: 26 July 2017
} 

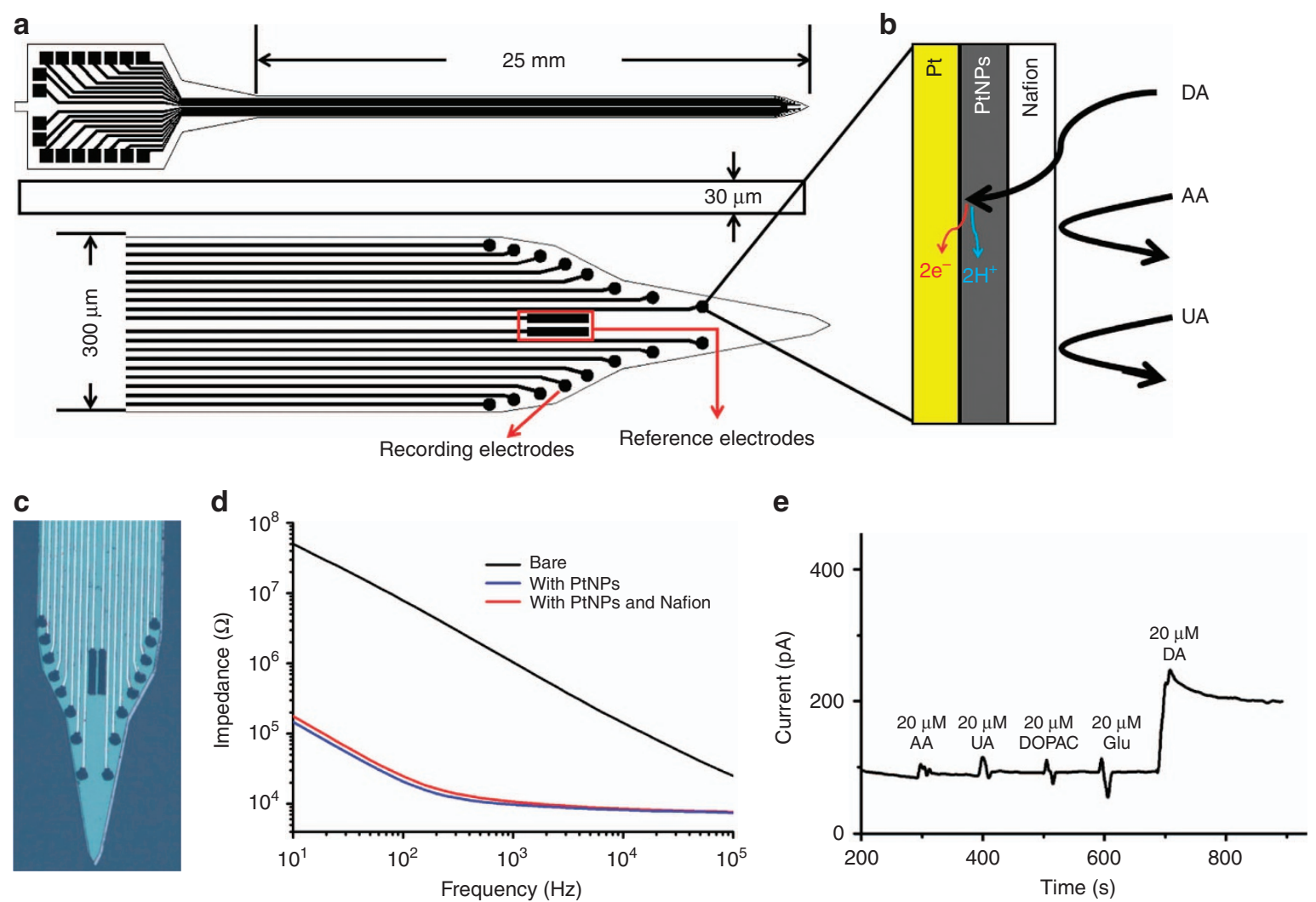

Figure 1 The implantable microelectrode array (MEA) probe is created using silicon-on-insulator (SOI) substrates via the microelectromechanical systems (MEMS) method. (a) The MEA silicon probe is designed and fabricated like a needle, and the total length of the implantable part is set as $25 \mathrm{~mm}$. (b) To decrease the electrode impedance and improve the signal-to-noise ratio, Pt nanoparticles (PtNPs) are electroplated onto the bare microelectrodes. After deposition of the PtNPs, the ion-exchange resin Nafion is drop-coated onto the probe tips. (c) Photomicrograph of the MEA after deposition of the PtNPs and Nafion. (d) Impedance spectra of microelectrodes before and after PtNPs and Nafion are deposited onto them. (e) Typical amperometric responses of interferents in the online detection system at a working potential of $+500 \mathrm{mV}$. The AA $(20 \mu \mathrm{m})$, UA $(20 \mu \mathrm{m})$, DOPAC $(20 \mu \mathrm{m})$ and Glu $(20 \mu \mathrm{m})$ interferents are added into the PBS to obtain the selectivity. AA, ascorbic acid; UA, uric acid; DOPAC, dihydroxyphenylacetic acid; Glu, glutamic acid; DA, dopamine.

Early reports showed that HFS increased major DA metabolic products in the striatum of 6-hydroxydopamine (6-OHDA) lesioned rats ${ }^{15,16}$. This result could explain the immediate improvement after the electrical stimulation. However, the rat PD animal models induced by 6-OHDA are markedly different from humans with PD. There are limitations to the clinical research.

Actually, far more complex and wide-ranging impacts are induced in this dynamical neural network. Multilevel intensive research is still indispensable. Since the neuronal communications are inherently electrical and chemical in nature, observation of the neural electrophysiological features and neurotransmitter levels together in affected brain areas during PD progress is considered an important method. However, the connections between the chemical transmitters and electrical activities are still not well understood in vivo. Therefore, dual-mode detection of PD-related neuroelectrical and neurochemical information is essential for pathologic research, early diagnosis, therapeutic effect assessments, and new treatment developments of the disease ${ }^{17,18}$.

Previous neural information detections in parkinsonian animal models were based on traditional implantable devices. In neuroelectrical studies, sharpened metal wire microelectrodes and glass micropipettes have been used to record single-unit neural spikes in the cortices and striata of parkinsonian animals. In neurochemical studies, microdialysis probes and carbon fiber electrodes have been widely used for the neurochemical recording of the DA concentration in multiple brain sites $8,19,20$. With the development of microelectromechanical systems (MEMS) technology, silicon-based microelectrode arrays (MEA) deposited on micro probes have been increasingly shown to be effective tools for multichannel neural information detection with high spatial resolution ${ }^{21-24}$. Michigan-style probes have been successfully used in a number of neuroscience applications, but they suffer from some disadvantages related to their limited thickness and length ${ }^{25}$. In recent years, micromachined silicon probes with monolithically integrated microfluidic channels of up to $15-70 \mathrm{~mm}$ in length have been realized to perform simultaneous electrical recording and drug delivery in deep brain regions ${ }^{26}$. Such silicon-based probes have promising application prospects in the field of neuroscience; however, presently, few studies have provided insights into their dual-mode detection performance in non-human primate models. To achieve these goals, new MEMS probes should be explored.

For this purpose, we describe a silicon-based, 16-site implantable MEA, fabricated using MEMS technologies, for in vivo testing. The probe is $25 \mathrm{~mm}$ long and designed specifically for use in nonhuman primates for electrophysiological and electrochemical recording. In previous experiments, we have shown that the MEA probe can be successfully used in non-human primates to record neural action potentials (spikes), local field potentials (LFPs), and DA variation continuously from the cortex to the striatum. In this paper, to evaluate its applicability to PD research, the neuroelectrical and neurochemical detections are performed in both normal and hemiparkinsonian monkey models. Multichannel data of spikes and LFPs, as well as DA oxidation currents, are simultaneously acquired and deeply analyzed. The dual-mode MEA probe is demonstrated to be an effective tool for neurological disorder research. 


\section{MATERIALS AND METHODS}

\section{Reagents and apparatus}

The reagents, that is, urethane $\left(\mathrm{C}_{3} \mathrm{H}_{7} \mathrm{NO}_{2}\right)$, lead acetate $(\mathrm{Pb}$ $\left.\left(\mathrm{CH}_{3} \mathrm{COO}\right)_{2}\right)$ and chloroplatinic acid $\left(\mathrm{H}_{2} \mathrm{PtCl}_{6}\right)$, were purchased from Sinopharm Chemical Regent (Beijing, China). Dopamine hydrochloride was purchased from Acros Organics (Belgium, USA). Nafion perfluorinated ion-exchange resin was purchased from Sigma Aldrich (Billerica, MA, USA). Saline $(0.9 \% \mathrm{NaCl})$ was obtained from the ShuangHe Corporation (China). We prepared phosphatebuffered saline (PBS, $0.1 \mathrm{M}, \mathrm{pH}$ 7.4) from a PBS tablet (Sigma, Billerica, MA, USA) with deionized (DI) water. All solutions were prepared in DI water, which was purified using an MW-D20 system (MeiCheng, resistivity $>18 \mathrm{M} \Omega$ ).

All electrochemical measurements were carried out using a potentiostat (Gamry Reference 600, Warminster, PA, USA). The electrical signals were measured using a 128-channel neuron data recording system (Blackrock Microsystems, Salt Lake City, UT, USA). A micropositioner (model 2662, David KOPF Instruments, Tujunga, CA, USA) was used to implant the MEA probe. Other apparatuses included an ultrasonic generator (KH2200E, Hechuang, China), stereotaxic frame (Stenting, Wood Dale, IL, USA), and optical microscope (BX51TRF, Olympus, Japan).

\section{MEA probe fabrication and modification}

The recording electrodes used in our studies are specifically designed with a long shank that allows for a total length of $25 \mathrm{~mm}$ to penetrate the depth of the primate brain, as shown in Figure $1 \mathrm{a}$. The MEA silicon probe consists of a single shank and has a crosssection of $30 \mu \mathrm{m} \times 300 \mu \mathrm{m}$. Along the shank, there is a v-shaped array of 16 round microelectrode sites ( $20 \mu \mathrm{m}$ in diameter) on the tip of the probe, and each site can be selected for neuroelectrical or neurochemical detection. The center-to-center site separation is 50-100 $\mu \mathrm{m}$ between two adjacent sites. Maintaining that distance between recording sites is reasonable, as a shorter interelectrode separation can result in possible crosstalk artifacts, whereas a larger separation can result in missed cross-correlations between neuron pairs. Two rectangular electrodes $(25 \mu \mathrm{m} \times 300 \mu \mathrm{m})$ are integrated on the probe as the optional reference or auxiliary electrodes. Connecting lines run from these contacts to the distal end of the device tip and terminate in bonding pads.

The improved MEA was mass fabricated in our clean room following the same process as used for our previously described probe ${ }^{27}$ : (1) $\mathrm{A} \mathrm{SiO}_{2}(500 \mathrm{~nm})$ insulator was grown on the wafer via thermal oxidation. (2) $\mathrm{Ti} / \mathrm{Pt}(30 \mathrm{~nm} / 250 \mathrm{~nm})$ was sputtered and patterned via a photoresist lift-off process to form a conducting layer of the microelectrodes and the leads on top of the silicon surface. (3) $\mathrm{A} \mathrm{SiO}_{2} / \mathrm{Si}_{3} \mathrm{~N}_{4}(300 \mathrm{~nm} / 500 \mathrm{~nm})$ passivation layer was deposited via plasma-enhanced chemical vapor deposition and then selectively removed via $\mathrm{SF}_{6}$ reactive ion etching using a resist mask to uncover the microelectrode sites and the bonding pads. (4) The top silicon layer $(30 \mu \mathrm{m})$ was selectively etched down to the buried oxide using inductively coupled plasma deep reactive ion etching with a resist mask. (5) With the top side of the silicon protected by melting asphaltum, the back side of the silicon was wet etched in a $\mathrm{KOH}$ solution (the crystal orientation was $<100\rangle, 50 \%, 80^{\circ} \mathrm{C}$ ) until self-stopped, and then the buried oxide $(2 \mu \mathrm{m})$ was removed in HF buffer. (6) The probes were released by dissolving the top side of the asphaltum in developing liquid. After ultrasonic cleaning, the probes were assembled onto interfacing printed circuit boards via wire bonding.

The electrode impedance affects the neural signal detection. Detecting neural signals is a function of the geometry and surface area of the exposed recording surface, as well as the materials used for recording ${ }^{28}$. To decrease the electrode impedance and improve the signal-to-noise (SNR) ratio, all recording sites were modified using Pt nanoparticles (PtNPs). This layer enlarged the recording surface area and decreased the site impedance. PtNPs were electrodeposited onto the round microelectrodes using chloroplatinic acid $(48 \mathrm{~mm})$ and lead acetate $(4.2 \mathrm{~mm})$ in a 1:1 mixed solution at $-1.1 \mathrm{~V}$ using a Pt electrode for $30 \mathrm{~s}$. After the PtNPs had been deposited, the ion-exchange resin Nafion (1\% in ethanol) was drop-coated onto the probe tips and dried for $20 \mathrm{~min}$ at $80^{\circ} \mathrm{C}$. The Nafion film can block ascorbic acid (AA), uric acid (UA), dihydroxy-phenyl aceticacid (DOPAC) and glutamate (Glu) from reaching the $\mathrm{Pt}$ recording sites to improve the selectivity for DA detection (Figure 1b) ${ }^{29,30}$. Figure $1 \mathrm{c}$ shows a photomicrograph of the MEA probe tip.

\section{Electrochemical characterization}

To further characterize the performance of the MEA probe modified using PtNPs and Nafion, prior-implant calibrations were used to assess the in vivo DA concentrations. Calibrations were carried out in standard PBS solutions. An $\mathrm{Ag} \mid \mathrm{AgCl}$ electrode was used as the reference electrode. The sensitivity was determined via a stepwise addition of 20 to $160 \mu \mathrm{m}$ of DA hydrochloride solutions (in saline) to the buffer. The current-time (I-t) curve was continuously recorded for each microelectrode. A voltage of $+0.5 \mathrm{~V}$ versus the $\mathrm{Ag} \mid \mathrm{AgCl}$ reference was applied to the working electrode. All calibrations were carried out at room temperature. DA is a kind of electroactive substance. It does not need enzymes to be involved in the oxidation process. When sufficient potential is applied to the electrode, DA is oxidized to dopamine-o-quinone, donating two electrons that are detected as a current. The DA level is proportional to the current.

\section{In vivo experiments}

Our group was able to repeat the experiment in animals at multiple time points spaced weeks and months apart. The same animal could be used for both normal and post-MPTP treatment analyses. Behavioral measurements carried out on these animals demonstrated that the surgical procedures produced only modest transitory behavioral changes in these animals. Therefore, we recorded the dual-model neural signals of the normal monkey first as a basic reference. Then, the monkey was administered an MPTP injection via the carotid artery unilaterally. After stable parkinsonian features had developed, the same measurements were carried out under the same conditions as before.

In acute implantation surgery, the experimental monkey was not allowed to consume food or water the night before the surgery. Anesthesia was induced using isoflurane gas (1-3\%) following a preoperative dose of ketamine hydrochloride (10$\left.30 \mathrm{mg} \mathrm{kg}^{-1}, \mathrm{IM}\right)$ and atropine $\left(0.04 \mathrm{mg} \mathrm{kg}^{-1}, \mathrm{IM}\right)$. Animals were intubated and placed in a compatible stereotaxic frame. A craniotomy was performed using the coordinates of the rectangular cranial with respect to the bregma. Areas of the skull overlying the caudate nucleus and striatum were removed, which exposed a section of the cortical surface. The overlying meninge was removed to expose the surface of the brain for penetration of the probe. An $\mathrm{Ag} \mid \mathrm{AgCl}$ wire reference electrode was placed between the dura mater and the skull. A stainless-steel bone screw was twisted into the skull to establish a ground connection. The MEA probe was implanted according to the stereotaxic atlas of the monkey brain, and the stimulating electrodes (outer diameter: $200 \mu \mathrm{m}$, CEA-200, Microprobes Inc., Gaithersburg, MD, USA) were also implanted in the STN of the lesioned side at the same time. Using a motor-driven hydraulic propeller, the MEA was inserted successively across the cortex and the striatum at a constant speed. The entire experiment was conducted in a Faraday cage.

The implanted MEA probe was connected to the electrophysiological recording system and the electrochemical workstation. For electrophysiological detection, eight microelectrode sites were selected and connected to a 128-channel data acquisition system. The 8-channel raw data were acquired simultaneously and 
continuously with a sample rate of $30 \mathrm{kHz}$. A bandpass filter (250$5 \mathrm{kHz}$ ) was applied to extract neural action potentials. A low-pass filter $(0-250 \mathrm{~Hz})$ was used to extract LFPs. For neurochemical detection, a site was selected and connected to the single-channel potentiostat. The DA signals were recorded using constant amperometry responses. The working potential was held at $+0.5 \mathrm{~V}$ versus the $\mathrm{Ag} \mid \mathrm{AgCl}$ reference wire, and signals were recorded at $0.1 \mathrm{~s}$ intervals.

All animal experiments were performed in a fully AAALACaccredited primate facility (Wincon TheraCells Biotechnologies Co., Ltd., Nanning, China), with the temperature of the feeding room being maintained from 22 to $28^{\circ} \mathrm{C}$, the relative humidity being in the range from 30 to $75 \%$, an alternating $12 \mathrm{~h}$ light/dark cycle, and an uninterrupted water supply ad libitum. Animals were fed twice daily, with an additional portion consisting of fresh fruits and vegetables being offered once daily at noon.

\section{RESULTS AND DISCUSSION}

\section{Impedance and selectivity test of the electrodes}

Figure $1 \mathrm{~d}$ details the electrochemical impedance spectrum of the microelectrodes before and after the PtNPs are deposited onto them. The nanoparticles possess large surface areas and therefore increase the geometrical surface area of the electrode, thus reducing the electrode impedance. The average impedance of the bare microelectrodes at $1 \mathrm{kHz}$ is $1.17 \pm 0.15 \mathrm{M} \Omega(n=16)$. After coating the electrodes with PtNPs, the mean impedance decreases to $9.80 \pm 1.8 \mathrm{k} \Omega(n=16)$. In addition, after being modified with PtNPs and Nafion, the mean impedance increases to $11.0 \pm 2.2 \mathrm{k} \Omega$ $(n=16)$, which is a desirable range for electrical neural recording. Decreased impedance provides improved performances, that is, LFP and single-unit recordings with lower noise, increases the number of detectable neural units, and reduces the amount of recording artifacts and noise.

Figure 1e shows the typical amperometric responses of interferents in the online detection system at a working potential of $+0.5 \mathrm{~V}$. AA $(20 \mu \mathrm{m})$, UA $(20 \mu \mathrm{m})$, DOPAC $(20 \mu \mathrm{m})$ and Glu $(20 \mu \mathrm{m})$ are added into the PBS to obtain the sensor selectivity. The results demonstrate that the interference conditions cannot produce measurable responses in the PtNPs-Nafion-modified channel, which convincingly indicates that the DA measurements are essentially interference-free with respect to these electroactive species.

\section{Amperometric response for the dopamine MEA probe}

Prior to the surgical procedures, each PtNPs-Nafion-modified recording site was characterized based on its sensitivity to DA. In the DA solution, within the scanning potential range of $-0.1 \sim+0.8 \mathrm{~V}$, there was an oxidation peak current on the CV curve. The peak potential was $+0.5 \mathrm{~V}$. This result indicates that the DA oxidized under this potential. A typical current-time response curve of one electrochemical site for different additions is shown in Figure 2a. A voltage of $+0.5 \mathrm{~V}$ (maximum oxidation voltage) versus the $\mathrm{Ag} \mid \mathrm{AgCl}$ reference was applied to the working electrode. After $400 \mathrm{~s}$, the baseline was unchanged and exhibited a background current of $100 \pm 3 \mathrm{pA}$. As demonstrated, the electrochemical sites were stable enough to respond to a low current change. A calibration plot of the DA concentration in the range of $20-160 \mu \mathrm{m}$ is shown in Figure $2 \mathrm{~b}$. The microelectrode exhibited a sensitivity of $13.8 \mathrm{pA} \mu \mathrm{M}^{-1}$ to DA and had a relative coefficient of 0.999 . For the other seven microelectrodes, although with different baseline currents, their sensitivities were nearly the same, and the average value was $13.5 \pm 0.4 \mathrm{pA} / \mu \mathrm{m}(n=6)$. The calibration results show that all sites can be reliably applied during in vivo recording.
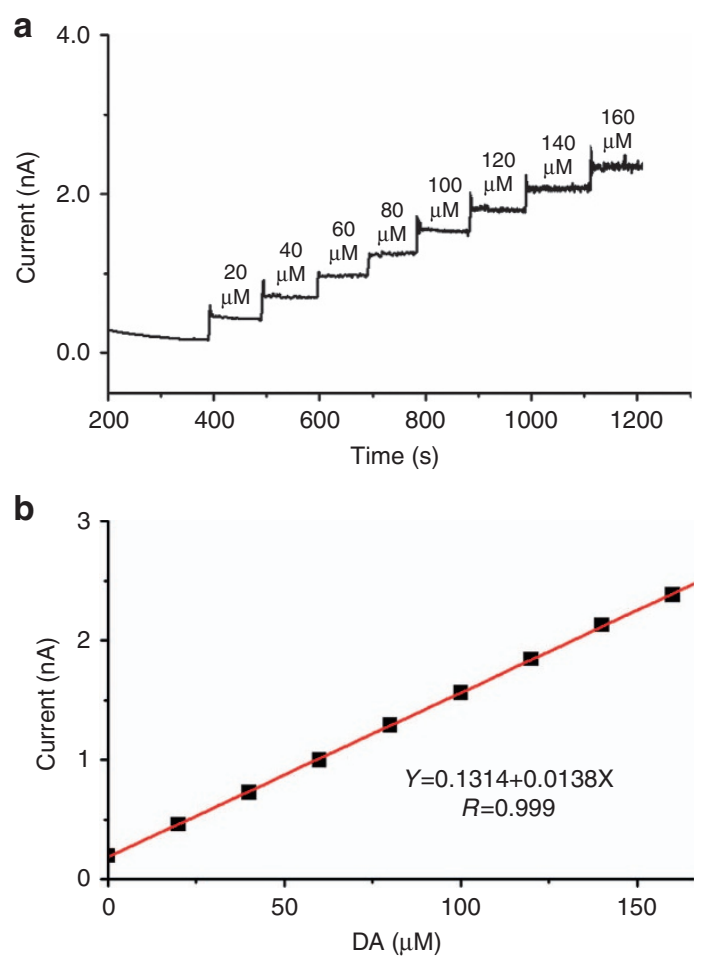

Figure 2 The sensitivity to dopamine (DA) and linear range of the recording sites modified using $\mathrm{Pt}$ nanoparticles (PtNPs) and Nafion. (a) Current-time response of the microelectrode array (MEA) to varying concentrations of DA. The applied potential was held at $+0.5 \mathrm{~V}$ vs. AglAgCl. (b) Resulting linear plot of the DA response vs. DA concentration of the MEA probe. The sensitivity is $13.8 \mathrm{pA} \mathrm{\mu M}^{-1}$, $R=0.999$.

\section{Dopamine-related neurochemical analysis}

PD patients express motor symptoms only after $60-80 \%$ striatal DA depletion. In hemiparkinsonian animal models, dopaminergic nigral neurons are reduced by more than $85 \%$ in the substantia nigra and corpora striata of the lesioned side compared with the unlesioned side according to a semiquantitative analysis ${ }^{31,32}$.

In previous studies, our team mainly focused on the dynamic feature of DA in normal monkeys. Such studies demonstrated that the dynamic change in DA overflow could be investigated with rapid electrochemical recordings using our MEA probes. We showed the inhomogeneous distribution of DA from the cortex to the striatum. In particular, the content of DA in the striatum was much larger than that in the cortex. In this study, we continue to research the neural information of PD monkey models. The primary target is to analyze the dynamic evaluation of DA change in the striatum of non-human primates.

First, according to our experiment plan, we recorded the dualmodel neural signals of the normal monkey as a base reference. The MEA probe was inserted through the cortex into the deep brain of the striatum at a constant velocity $\left(10 \mu \mathrm{m} \mathrm{s}^{-1}\right)$. The $I-t$ curve was recorded continuously throughout this process. After that, the normal monkey was administered an MPTP injection via the carotid artery unilaterally. When stable parkinsonian features had developed, the above process was repeated. In Figure $3 a$, the blue line shows the amperometric l-t curve recorded by Ch5 during the continuous inserting of the MEA probe in the normal monkey, while the red line represents the current curve after the PD model was established. From the results, we found significant differences between these two experiments.

For the normal monkey, there was a prominent rise of the current line during the inserting process. One microelectrode site (Ch5) was selected for neurochemical recording. At the beginning 

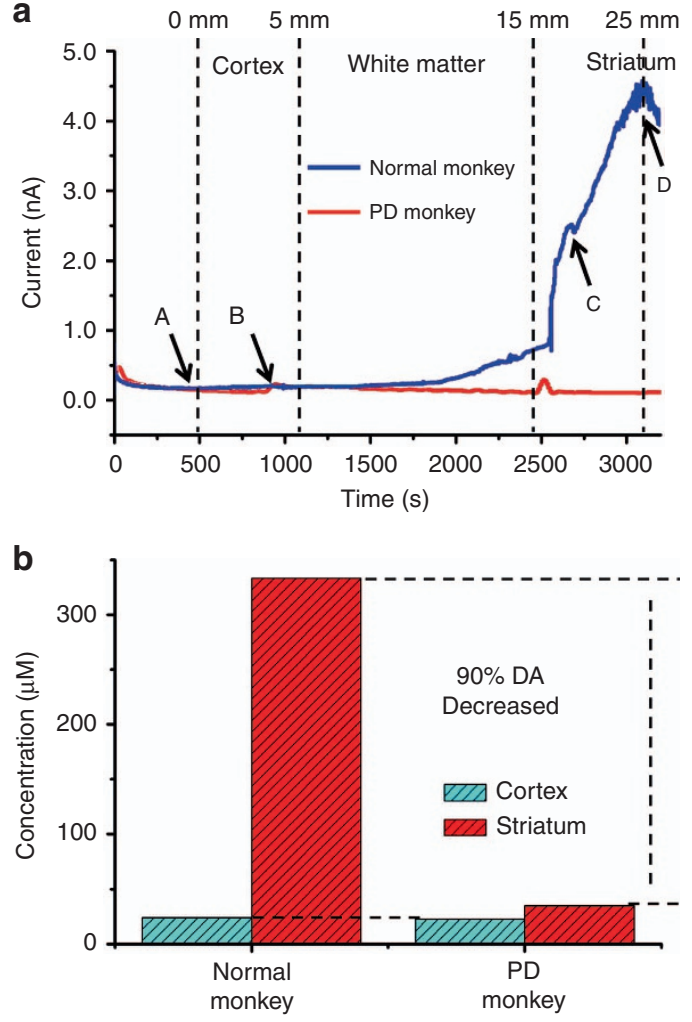

Figure 3 The DA levels recorded during the continuous insertion of the microelectrode array (MEA) probe from the cortex into the striatum. (a) The dynamic change in dopamine (DA)overflow from the cortex to the striatum, investigated via rapid electrochemical recordings using an MEA probe. The blue line represents the normal monkey, while the red line represents the Parkinson's disease (PD) monkey. (b) The histograms of the DA levels in the cortex and striatum of both the normal monkey and PD monkey.

of insertion, the MEA probe was in contact only with the surface of the brain. After recording in a static state for approximately $500 \mathrm{~s}$ $\left(0 \mu \mathrm{m} \mathrm{s}^{-1}\right)$, the current gradually decreased to $215 \mathrm{pA}$ (depth $=0 \mathrm{~mm}$ ) and remained stable (point $A$ ). Then, we advanced the electrode to the cortex, and the current gradually increased up to $330 \mathrm{pA}$ (depth $=4.5 \mathrm{~mm}$, point $\mathrm{B}$ ). This result suggested that there was only a low concentration of DA in the cortex. When the MEA passed through the cortex and white matter to the striatum, the oxidation current rapidly increased to $2.43 \mathrm{nA}$ (point C). We continued propelling the MEA until it reached its maximum (depth $=25 \mathrm{~mm}$, point $\mathrm{D}$ ), and the current continued to rise, reaching a peak of $4.59 \mathrm{nA}$. The change in the current throughout this process reflected the inhomogeneous distribution of DA in the striatum.

In contrast, for the MPTP-treated monkey, the current line remained nearly unchanged throughout the whole process. After recording for $\sim 500 \mathrm{~s}\left(0 \mu \mathrm{m} \mathrm{s}^{-1}\right)$, the current gradually decreased to $220 \mathrm{pA}$ and held steady. Then, the current gradually increased to $310 \mathrm{pA}$ (depth $=4.5 \mathrm{~mm}$, point $B$ ) when the probe reached the cortex. As the MEA probe approached the position of the striatum (depth $\geq 15 \mathrm{~mm}$ ), no increase in the current curve was observed (the maximum value was $480 \mathrm{pA}$ ). It remained 'silent' during the whole course. This phenomenon indicated that the DA concentration in the striatum severely decreased after the model had been established. The peak at $2500 \mathrm{~s}$ indicated that the electrode had just penetrated into the striatum.

In Figure 3b, the DA levels in the cortex and striatum of both the normal and PD monkeys are plotted as histograms. The DA content in the cortex of both the normal monkey and the PD monkey was $\sim 23.2 \mu \mathrm{m}$. This result indicates that there was only a low concentration of DA in the cortex. In the striatum, the DA content was $\sim 332.6 \mu \mathrm{m}$ for the normal monkey and $35.2 \mu \mathrm{m}$ for the PD monkey. A 90\% decrease in the DA level was found in the striatum after the PD model had been established. This decrease was caused by the degeneration of dopaminergic neurons of the substantia nigra pars compacta, which led to severe dopaminergic denervation of the corpus striatum.

\section{Analysis of dual-mode signals}

Activities in the nervous system include a complex combination of biochemical and electrical events in space and time. The local field potential integrates predominantly synaptic input signals from a large population of neurons, whereas action potentials are the output signals of a single neuron. Thus, investigating the electrophysiological signals corresponding to these activities may enable a better understanding of dopaminergic processes. In our experiments, the neural signals were recorded while the probe was inserted into deeper brain regions. Electrophysiological signals were continuously recorded at eight sites during simultaneous DA detection. The neuron discharge style and patterns of spikes were analyzed.

Through our experiment, high-quality electrophysiological signals were obtained, and several channels often had multiple waveforms of different amplitudes and shapes. Across any given microelectrode, the noise floors were $20 \mu \mathrm{V}$, and spike amplitudes ranged from 70 to $360 \mu \mathrm{V}$ peak to peak. The average SNR of each electrode was $>5$.

Figure $4 a$ shows examples of spike trains and the simultaneously recorded LFP in the normal and PD monkey. The recording locations in these two experiments were the same. For each monkey, the electrical signals were recorded separately at depths of $4.50 \mathrm{~mm}$ (cortex), $9.90 \mathrm{~mm}$ (white matter) and $17.00 \mathrm{~mm}$ (striatum) for comparison.

When the MEA was inserted into the cortex of the normal and PD monkeys, small current peaks with respect to the baseline were observed for both. This result suggested that there was only a low concentration of DA in the cortex. The spikes and LFPs recorded at the corresponding electrical sites were analyzed as follows.

In the cortex of the normal monkey, the amplitude of spikes was large, but the frequency was low. The firing pattern of the spikes was mainly scattered. However, after the PD model had been established, the spike trains exhibited different characteristics compared to the previous situation: the fire frequency of the spikes improved significantly. In addition, the firing pattern of the spike train exhibited dense activity. Compared to the normal monkey, the spikes recorded in the PD model fired more intensively. Additionally, burst patterns, which were not observed for the cortex of the normal monkey, began to appear. As we know, the cortex is the main region in control of cerebral functions such as movement and sensation. After the non-human primate developed PD, the neural cells in the cortex became more active.

The cortex and the striatum are two important brain regions involved in the progress of PD, and sophisticated motor control circuits connect these two regions. The striatum is a complex tissue as a result of its many subregions. Therefore, when the MEA passed through the cortex and the white matter to reach the striatum, marked changes were detectable in both the electrochemical and electrophysiological signals.

In the striatum of the normal monkey, the oxidation current quickly increased. The DA in the striatum of the healthy monkey was abundant. The amplitude of the spikes in the striatum was much smaller than in the cortex and white matter, but the firing rate was much higher. The firing pattern of the spikes train exhibited almost dense activity. After the PD model was established, the DA level and cellular activities were greatly 

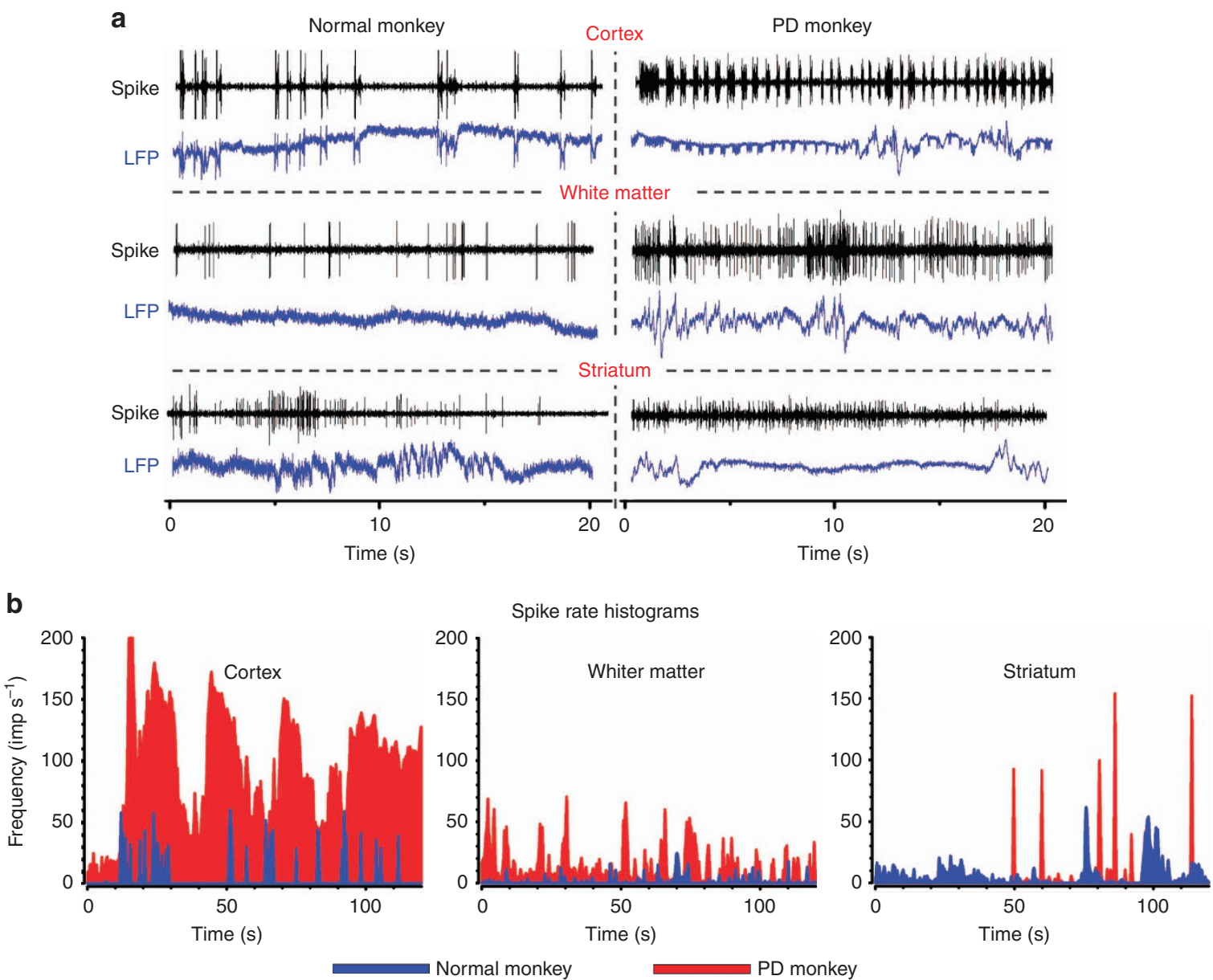

Figure 4 The typical signal distribution of three different locations in the normal monkey and Parkinson's disease (PD) monkey. (a) Examples of spike trains and simultaneously recorded local field potential (LFP) in the normal and PD monkeys. During the same $20 \mathrm{~s}$ time segments, the spike trains were different with respect to the amplitude and firing rate. The neural cells were more active in the PD monkey. (b) The spike rate histograms of the cortex, white matter and striatum. The blue curves represent the spike firing rate of the normal monkey, while the red curves represent that of the PD monkey.

reduced. As we know, PD is characterized by a striatal DA deficit due to a progressive loss of nigrostriatal DA neurons. We assumed that these changes in nerve cell activities might be related to the lack of DA.

Figure $4 \mathrm{~b}$ shows the spike rate histograms of the cortex, white matter, and striatum. The coordinate systems of the three figures were all chosen to be the same. In the pictures, the blue curves denote the spike firing rate of the normal healthy monkey, while the red curves denote the spike firing rate of the PD monkey. The results show that after suffering from $P D$, the spike firing frequency of these three regions increased significantly. This result indicates that the neuronal cells became even more active than normal, especially in the cortex.

\section{Analysis of LFP signals}

The LFP is defined as the low-frequency (below $250 \mathrm{~Hz}$ ) component of the raw field potential. Therefore, the vertical axes in Figure 5a display only frequencies below $30 \mathrm{~Hz}$. To facilitate the analysis, we selected three different depths: $4.50 \mathrm{~mm}$ (cortex), $9.90 \mathrm{~mm}$ (whiter matter) and $17.00 \mathrm{~mm}$ (striatum), just as before. Figure 5a shows the spectrogram analysis of these three different locations in both the normal and PD monkeys.

Our main result was that the LFP power spectrogram changed in the frequency band of $0-30 \mathrm{~Hz}$ after the normal monkey developed PD. As observed by comparing the power spectrograms of the normal and PD monkeys, the energy distributions were very different. For the normal monkey, the energy was mainly concentrated in the frequency range of $0-2 \mathrm{~Hz}$. It exhibited slow-wave activity. However, for the PD monkey, the spectrogram analysis showed that much greater power existed in the frequency band of $0-10 \mathrm{~Hz}$ compared to that of the normal monkey. The high-frequency spikes may have impacted the low-frequency LFPs in the frequency domain. They increased the power of the LFPs. The greater the number of spikes recorded, the larger the energy acquired. The frequency-dependent difference in the LFP power spectrogram may be related to severe dopaminergic denervation of the brain.

Figure $5 \mathrm{~b}$ presents an analysis of the power spectral density (PSD) of the LFPs carried out to obtain knowledge regarding their features in the frequency domain. We put the data in the same coordinate systems and analyzed their relative features. Although the depths were different, the features were very similar. In the normal monkey, the PSD was concentrated below a frequency of $2 \mathrm{~Hz}$. The peak values for the relative PSD were $\sim 30 \%$. However, for the PD monkey, the peak values of the PSD curves shifted to the right. The peak values for the relative PSD were $\sim 10 \%$. There was a reduction in the frequency range of $0-2 \mathrm{~Hz}$ and a distinct increase in the frequency range of $2-8 \mathrm{~Hz}$.

\section{HFS of STN in the hemi-parkinsonian monkeys}

The aim of this experiment was to examine the effects of STN-HFS on the DA level in the striatum of a hemiparkinsonian monkey. 


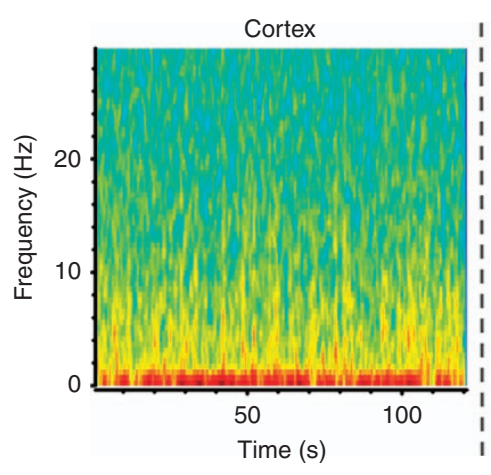

Spectrogram analysis
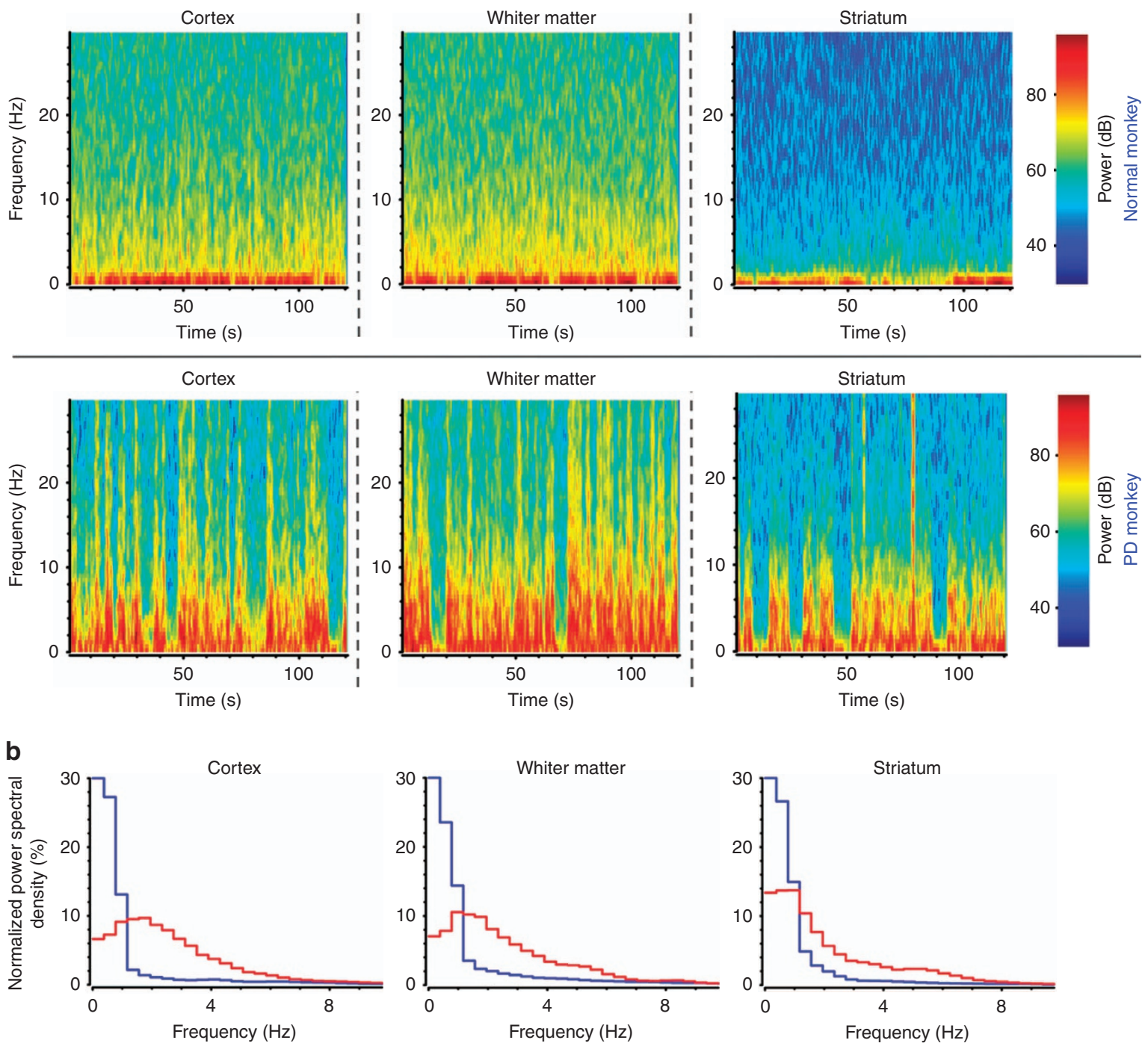

Normal monkey

PD monkey

Figure 5 Power spectrum analysis and power spectral density (PSD) of local field potential (LFP) in both normal and Parknison's disease (PD) monkeys. (a) The spectrogram analysis of three different locations in both normal and PD monkeys. For the normal monkey, the energy is mainly concentrated in the frequency range of $0-2 \mathrm{~Hz}$. For the PD monkey, the spectrogram analysis shows that much greater power existed in the frequency band of $0-10 \mathrm{~Hz}$. (b) PSD of three locations and their relative features.

The change in DA concentration in the corpus striatum was observed by our MEA probe in vivo. The stimulating electrode was implanted in the STN of the lesioned side before the recording. The STN was stimulated using a bipolar electrode with a biphasic pulse train triggered by a multichannel base station (MC Technologies, Germany) and delivered via a stimulus isolator.

At the beginning of the experiment, we inserted the MEA probe through the cortex into the striatum with a nearly constant velocity of $10 \mu \mathrm{m} \mathrm{s}^{-1}$ (point A to B in Figure 6; A: depth $=0 \mathrm{~mm}, \mathrm{~B}$ : depth $=17 \mathrm{~mm}$ ). The $l-t$ curve was recorded continuously throughout this process. We found that the DA oxidation current remained nearly unchanged throughout this process. This result shows that the DA content was severely decreased in the striatum, as mentioned before. Then, the pulse generator was turned on, and the stimulation parameters were set to those used in clinical practice $(100 \mathrm{~Hz}$ frequency, $60 \mu \mathrm{s}$ rectangular pulse width and $200 \mu \mathrm{A}$ intensity).
A representative example of this recording is shown in Figure 6. DA overflow in the striatum was observed following electrical stimulation. After repeated STN stimulation trials, the DA current in the striatum increased from 242 to $470 \mathrm{pA}$ gradually within $30 \mathrm{~min}$ and then remained in dynamic balance (point C). The electrical stimulation resulted in electrochemically transduced DA transients in the striatum that were spatially heterogeneous within at least $16.5 \mu \mathrm{m}$. This phenomenon showed that the levels of DA in the striatum of the electrode side were increased as a result of STN-HFS. The symptom improvement of the hemiparkinsonian monkeys correlated with the increase in DA in the corpus striatum. This study led to a better elucidation of the mechanism of STNHFS treatments.

\section{Stability of the MEA}

An ideal array is one that can record high-quality signals from the same set of cells for a long time. In our studies, acute recordings 


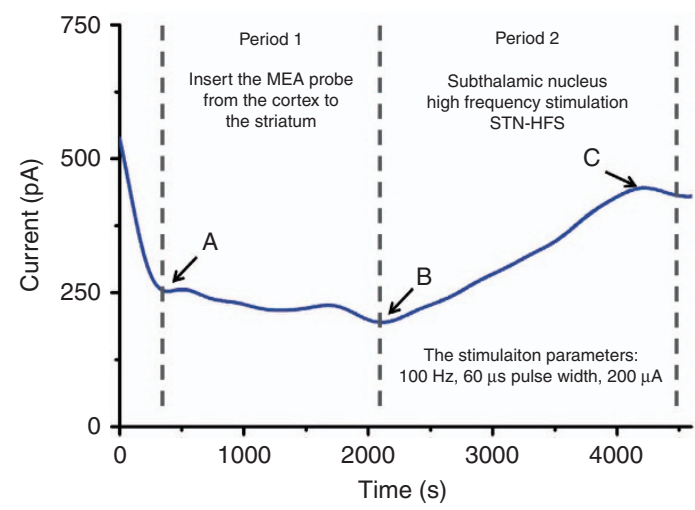

Figure 6 The amperometric $l-t$ curve recorded in the striatum of the hemiparkinsonian monkey before and during STN-HFS. The dopamine (DA) current increases from 242 to $470 \mathrm{pA}$ after STNHFS. The high-frequency stimulation (HFS) resulted in electrochemically transduced DA transients in the striatum of at least $16.5 \mu \mathrm{M}$. This change strongly suggests that STN-HFS enhances striatal DA release. MEA, microelectrode array.

were reliably obtained across the duration of our analysis (up to $10 \mathrm{~h}$ ) on the basis of both the number waveforms and quality of the signal obtained (for both the electrophysiological recording and dopamine sensing). The MEA was stored at $4{ }^{\circ} \mathrm{C}$ to examine the stability after completion of the modification. The MEA kept $95.2 \%$ of the initial response after a storage period of 2 months. The results revealed that the MEA exhibits good stability.

\section{CONCLUSIONS}

In the present study, we created a novel implantable MEA probe via MEMS methods that can be used for simultaneous measurements of DA, LFP and spike activities in normal and PD nonhuman primate brains. The electrochemical recordings proved that PD is caused by the death of dopaminergic neurons in the compact part of the nigra, which leads to severe DA reduction in the striatum. Because of the lack of DA, the neural cells in a brain became more active. The firing frequency of spikes of a PD monkey increased significantly, and the LFPs showed that much greater power existed in the $0-10 \mathrm{~Hz}$ frequency band. According to our experiment, the level of DA in the striatum was increased after STN-HFS. The beneficial effects of STN-HFS on PD symptoms are 'L-DOPA-like', but it remains unclear whether the mechanisms of action of STN-HFS and L-DOPA are similar or even synergic. The dual-mode MEA probe was demonstrated to be an effective tool for the study of neurological disorders. The next step would be to detect some neurotransmitters such as glutamate in a monkey brain.

\section{ACKNOWLEDGEMENTS}

This work was sponsored by the NSFC (Grant Nos. 61527815, 31500800, 61501426, 61471342), the National Key Research and Development Program (Grant No. 2017YFA0205900), the Beijing Science and Technology Plan (Grant Nos. Z141100000214002, Z1161100004916001), the National Science and Technology Major Project (2014CB744600), and the Key Programs of the Chinese Academy of Sciences (Grant Nos. KJZD-EW-L11-2, QYZDJ-SSW-SYS015).

\section{COMPETING INTERESTS}

The authors declare no conflict of interest.

\section{REFERENCES}

1 Gerhardt GA, Cass WA, Hudson J et al. In vivo electrochemical studies of dopamine overflow and clearance in the striatum of normal and mptp-treated rhesus monkeys. Journal of Neurochemistry 1996; 66: 579-588.

2 Hornykiewicz O, Kish SJ. Biochemical pathophysiology of Parkinson's disease. Advances in Neurology 1987; 45 (Suppl 2): 19.

3 Elsworth JD, Taylor JR Jr, Sladek JR et al. Striatal dopaminergic correlates of stable parkinsonism and degree of recovery in old-world primates one year after MPTP treatment. Neuroscience 1999; 95: 399-408.

4 Burns RS, Chiueh CC, Markey SP et al. A primate model of parkinsonism: Selective destruction of dopaminergic neurons in the pars compacta of the substantia nigra by N-methyl-4-phenyl-1,2,3,6-tetrahydropyridine. Proceedings of the National Academy of Sciences United States of America 1983; 80: 4546-4550.

5 Jenner P. The contribution of the MPTP-treated primate model to the development of new treatment strategies for Parkinson's disease. Parkinsonism \& Related Disorders 2003; 9: 131-137.

6 Neurology E. Rhythm-specific pharmacological modulation of subthalamic activity in Parkinson's disease. Experimental Neurology 2004; 189: 369.

7 Kühn AA, Williams D, Kupsch A et al. Event-related beta desynchronization in human subthalamic nucleus correlates with motor performance. Brain 2004; 127: 735.

8 Jankovic J. Movement disorders in 2016: Progress in Parkinson disease and other movement disorders. Nature Reviews Rheumatology 2017; 13: 76-78.

9 Zhao XD, Cao YQ, Liu HH et al. Long term high frequency stimulation of STN increases dopamine in the corpus striatum of hemiparkinsonian rhesus monkey. Brain Research 2009; 1286: 230-238.

10 Garris PA, Wightman RM. Different kinetics govern dopaminergic transmission in the amygdala, prefrontal cortex, and striatum: An in vivo voltammetric study. Journal of Neuroscience the Official Journal of the Society for Neuroscience 1994; 14: 442.

11 Justice JB Jr, Nicolaysen LC, Michael AC. Modeling the dopaminergic nerve terminal. Journal of Neuroscience Methods 1988; 22: 239-252.

12 Ray NJ, Brittain JS, Holland P et al. The role of the subthalamic nucleus in response inhibition: Evidence from local field potential recordings in the human subthalamic nucleus. Neuroimage 2012; 60: 271-278.

13 Abelson JL, Curtis GC, Sagher O et al. Deep brain stimulation for refractory obsessive-compulsive disorder. Biological Psychiatry 2005; 57: 510-516.

14 Koller W, Pahwa R, Busenbark K et al. High-frequency unilateral thalamic stimulation in the treatment of essential and parkinsonian tremor. Annals of Neurology 1997; 42: 292-299.

15 Meissner W, Harnack D, Reese R et al. High-frequency stimulation of the subthalamic nucleus enhances striatal dopamine release and metabolism in rats. Journal of Neurochemistry 2003; 85: 601-609.

16 Meissner W, Harnack D, Paul G et al. Deep brain stimulation of subthalamic neurons increases striatal dopamine metabolism and induces contralateral circling in freely moving 6-hydroxydopamine-lesioned rats. Neuroscience Letters 2002; 328: 105.

17 Disney AA, Mckinney C, Grissom L et al. A multi-site array for combined local electrochemistry and electrophysiology in the non-human primate brain. Journal of Neuroscience Methods 2011; 255: 29-37.

18 Johnson MD, Franklin RK, Gibson MD et al. Implantable microelectrode arrays for simultaneous electrophysiological and neurochemical recordings. Journal of Neuroscience Methods 2008; 174: 62-70.

19 Bruet N, Windels F, Bertrand A et al. High frequency stimulation of the subthalamic nucleus increases the extracellular contents of striatal dopamine in normal and partially dopaminergic denervated rats. Journal of neuropathology and experimental neurology 2001; 60: 15.

20 Degos B, Deniau JM, Chavez $M$ et al. Subthalamic nucleus high-frequency stimulation restores altered electrophysiological properties of cortical neurons in parkinsonian rat. PLOS ONE 2013; 8: e83608.

21 Lopez C, Andrei A, Mitra S et al. An implantable 455-active-electrode 52-channel CMOS neural probe solid-state circuits. IEEE Journal of Solid-State Circuits 2014; 49: 248-261.

22 Seidl K, Herwik S, Torfs T et al. CMOS-based high-density silicon microprobe arrays for electronic depth control in intracortical neural recording Microelectromechanical Systems. Journal of Microelectromechanical Systems 2011; 20: 1439-1448.

23 Scholvin J, Kinney J, Bernstein J et al. Close-packed silicon microelectrodes for scalable spatially oversampled neural recording. IEEE Transactions on Biomedical Engineering 2016; 63: 120-130.

24 Stephens ML, Pomerleau F, Huettl P et al. Real-time glutamate measurements in the putamen of awake rhesus monkeys using an enzyme-based human microelectrode array prototype. Journal of Neuroscience Methods 2010; 185: 264.

25 Wise KD, Najafi K. Microfabrication techniques for integrated sensors and microsystems. Science 1991; 254: 1335. 
26 Pongrácz A, Fekete Z, Márton G et al. Deep-brain silicon multielectrodes for simultaneous in vivo neural recording and drug delivery. Sensors \& Actuators B: Chemical 2013; 189: 97-105.

27 Zhang S, Song Y, Wang M et al. A silicon based implantable microelectrode array for electrophysiological and dopamine recording from cortex to striatum in the non-human primate brain. Biosensors \& Bioelectronics 2016; 85: 53.

28 Suner S, Fellows MR, Vargasirwin C et al. Reliability of signals from a chronically implanted, silicon-based electrode array in non-human primate primary motor cortex. IEEE Transactions on Neural Systems \& Rehabilitation Engineering 2005; 13: 524-541.

29 Phillips PEM, Wightman RM. Critical guidelines for validation of the selectivity of invivo chemical microsensors. Trac Trends in Analytical Chemistry 2003; 22: 509-514.

30 Erhan C, Erol A. Design of a multiwalled carbon nanotube-Nafion-cysteamine modified tyrosinase biosensor and its adaptation of dopamine determination. Analytical Biochemistry 2014; 444: 8-15.

31 Boulet S, Mounayar S, Poupard A et al. Behavioral recovery in MPTP-treated monkeys: Neurochemical mechanisms studied by intrastriatal microdialysis.
Journal of Neuroscience the Official Journal of the Society for Neuroscience 2008; 28: 9575-9584.

32 Lee $\mathrm{KH}$, Kristic K, Hoff RV et al. High-frequency stimulation of the subthalamic nucleus increases glutamate in the subthalamic nucleus of rats as demonstrated by in vivo enzyme-linked glutamate sensor. Brain research 2007; 1162: 121-129.

This work is licensed under a Creative Commons Attribution 4.0 International License. The images or other third party material in this article are included in the article's Creative Commons license, unless indicated otherwise in the credit line; if the material is not included under the Creative Commons license, users will need to obtain permission from the license holder to reproduce the material. To view a copy of this license, visit http://creativecommons.org/licenses/ by/4.0/

(c) The Author(s) 2018 